Research Paper

\title{
Immunohistochemical Detection of Human Papillomavirus 16 E7 Oncoprotein in Cervical Lesions
}

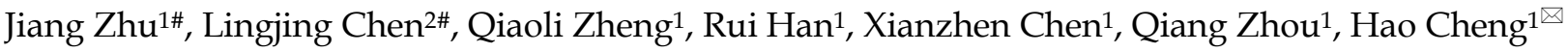 \\ 1. Department of Dermatology, Sir Run Run Shaw Hospital, School of Medicine, Zhejiang University, Hangzhou 310016, Zhejiang Province, China. \\ 2. Department of Dermatology, Hangzhou Children's Hospital, Hangzhou 310014, Zhejiang Province, China. \\ \#These authors contributed equally to this work. \\ $\square$ Corresponding author: Hao Cheng, MD, PhD, Professor. Phone: +86-571-86006975; Email: chenghao1@zju.edu.cn
}

(c) The author(s). This is an open access article distributed under the terms of the Creative Commons Attribution License (https://creativecommons.org/licenses/by/4.0/). See http://ivyspring.com/terms for full terms and conditions.

Received: 2021.03.17; Accepted: 2021.10.03; Published: 2021.10.20

\begin{abstract}
Almost all cervical cancer is associated with persistent infection of high-risk (hr) human papillomavirus (HPV) like HPV16. The E7 oncoprotein encoded by hrHPV plays a crucial role in carcinogenesis. The present study aimed to establish a reliable protocol of immunohistochemistry stains to detect HPV16 E7 protein in formalin-fixed and paraffin-embedded tissue specimens of various cervical lesions. Firstly, the HPV16 E7 gene was inserted into a prokaryotic expression vector PGEX-4T2. Then the recombinant plasmid PGEX-4T2-(HPV16-E7) was transformed into Escherichia coli JM109. The fusion protein containing a GST tag was purified, and New Zealand white rabbits were immunized to produce the HPV16 E7 polyclonal antibody. The anti-HPV16 E7 antibody was verified by western blotting and immunofluorescence stains, and applied in 182 HPV16 DNA-positive cervical tissue specimens and matched 36 HPV DNA-negative specimens by immunohistochemistry. Furthermore, A positive correlation between HPV16 E7 protein expression and malignancy grade was observed. But there is no relationship between HPV16 E7 protein expression and tumor sizes, tumor differentiation, lymph node metastasis, International Federation of Gynecology and Obstetrics (FIGO) stage, or lymphovascular space invasion in cervical cancer. These findings provide a basis for further research focusing on the role of HPV E7 protein in various HPV-related diseases.
\end{abstract}

Key words: Human papillomavirus (HPV), E7 protein, Polyclonal antibody, Immunohistochemistry, Cervical cancer

\section{Introduction}

Cervical cancer is the fourth most common cancer and the fourth leading cause of cancer death in women worldwide. According to the World Health Organization's global cancer observatory, there were an estimated 570,000 new cases and 311,000 deaths in 2018 worldwide. Besides, the incidence rate was 2 -fold higher, and the mortality rate was 3 -fold higher in low/medium Human Development Index (HDI) regions compared with high/very-high HDI regions [1], contributing to a massive public health burden, especially in low/medium HDI regions. The high-risk human papillomavirus (hrHPV) is a virtually necessary cause of cervical cancer, which could be detected in $99.7 \%$ of cervical cancer cases [2]. Persistent infection of hrHPV in the cervical epithelium was considered as a pivotal figure to cause cervical carcinogenesis [3], in which HPV16 and HPV18 had been classified as group 1 carcinogens by the IARC Monographs. It has been widely proved that early screening, diagnosis and treatment of HPV infection was an effective way to prevent cervical cancer.

The HPV genome consists of approximately 7,900 base pairs that can be divided into three regions: an early (E) region, a late (L) region, and a non-coding long control region containing regulatory elements for viral DNA replication and transcription. The L genes encode structural proteins. The $\mathrm{E}$ region contains six open reading frames (E1, E2, E4, E5, E6, E7) that are mainly for regulatory functions engaged in genome persistence [4], DNA replication [5], lytic cycle activation [6], and immune evasion [7]. The E6 and E7 
proteins as oncoproteins could interact with cell cycle checkpoints mechanisms by inactivating tumor suppressor proteins p53 and retinoblastoma protein to promote viral replication [8]. Besides, E6 and E7 proteins could interfere with the NF-kB pathway and interferon to escape immune detection [9]. Therefore, the E7 protein is one of the significant factors of HPV-associated carcinogenesis with markedly upregulated during transformation.

HPV genotyping analysis was widely used in clinical practice and provided great prevention against invasive cervical carcinomas [10]. But it could not distinguish from transient or persistent infection limits its predictive value. Therefore, oncoprotein E7 consistently expressed in HPV-transformed cervical cells could be a better diagnostic marker. The aim of this study was the expression of the HPV16 E7 protein in vitro, preparation of polyclonal anti-HPV16 E7 antibody, and its clinical application in various cervical lesions to investigate the relationship between HPV16 E7 protein expression and multiply clinical features.

\section{Materials and Methods}

\section{Patients, Animals and Cell lines}

This study was approved by the Ethics Committee of the Sir Run Run Shaw Hospital, College of Medicine, Zhejiang University and conducted with written informed consents of all participants. Formalin-fixed and paraffin-embedded cervical tissue specimens and their corresponding patient data were obtained from the Departments of Obstetrics and Gynecology, Sir Run Run Shaw Hospital, School of Medicine, Zhejiang University between 2009 and 2016 by cervical biopsy or hysterectomy. Patient data including tumor size, tumor differentiation grade, lymphovascular invasion, and lymph node metastasis was reviewed respectively. All patients were staged according to the International Federation of Gynecology and Obstetrics stage system, histologically classified and graded according to the WHO classification criteria, cytologically classified according to the Bethesda classification by two experienced pathologists. The inclusion criteria were described as follows: (1) polymerase chain reaction (PCR) proved HPV16 DNA-positive cervical lesions, (2) pathologists get consensus with histological classification, grade and cytological classification. The exclusion criteria were described as follows: (1) patients received chemotherapy, radiotherapy or immunotherapy before specimen collection. (2) tumor recurrence.

All animal procedures were performed in accordance with animal use protocols approved by
Zhejiang Chinese Medical University Animal Care and Use Committee and Zhejiang University Animal Care and Use Committee. New Zealand white rabbits ( 3 months old, weighing approximately $2.5 \mathrm{~kg}$ ) were supplied by the Laboratory Animal Research Center of Zhejiang Chinese Medical University (certificate number SYXK, Zhejiang, 2013-0184) to raise polyclonal antibodies. Human embryonic kidney (HEK) 293T cell line and human cervical squamous cell carcinoma $\mathrm{SiHa}$ cell line were cultured in Dulbeccos modified Eagles Medium (DMEM) supplemented with $4.5 \mathrm{~g} / \mathrm{L}$ glucose and $10 \%$ fetal bovine serum (FBS, Sijiqing, China).

\section{Plasmid Construction and Protein Purification of HPV16 E7}

The HPV16 E7 gene was amplified by PCR from HPV16 genomic DNA (ATCC, USA). The forward primer $\left(5^{\prime}\right.$ CCGGGATCCATGCATGGAGATACA CCTAC $3^{\prime}$ ) and the reverse primer (5' CCGGAA TTCTTATGGTTTCTGAGAACAGATG 3') containing $B a m \mathrm{HI}$ and EcoRI restriction enzyme sites were synthesized by Qingke Biological Technology Co., Ltd. (Zhejiang, China). The PCR product was cleaved with BamHI and EcoRI, purified and ligated to the BamHI- and EcoRI-digested pGEX-4T2 vector. pGEX-4T2-(HPV16-E7) encodes the HPV16 E7 protein containing a GST open reading frame. The Escherichia coli strain JM109 (Takara, Japan) was used as the host. The resultant plasmid was further confirmed to be correct by double restriction enzyme digestion and sequencing (Qingke Biological Technology Co., Ltd., Zhejiang, China).

The strains harboring HPV16 E7 were grown in LB medium containing $100 \mu \mathrm{g} / \mathrm{ml}$ ampicillin shaken at $250 \mathrm{rpm}$ and $37^{\circ} \mathrm{C}$ until the optical density (OD) $600 \mathrm{~nm}$ reached 0.6-0.8. Isopropyl $\beta-\mathrm{D}$ thiogalactopyranoside with a final concentration of $0.2 \mathrm{mM}$ was added, and the culture was incubated at $26^{\circ} \mathrm{C}$ for $4-6$ hours. Cells were harvested by centrifugation, resuspended in PBS, and sonicated on ice. The homogenate was centrifuged at 2,000 $\times \mathrm{g}$ for $5 \mathrm{~min}$, both supernatants and pellet fractions were collected. The fusion protein was purified by affinity chromatography with glutathione-Sepharose 4B beads (Life, USA), the GST-tag was removed by thrombin (Life, USA), the purified protein was dialysed against $\mathrm{PBS}$ overnight and stored at $-80^{\circ} \mathrm{C}$.

\section{Production and Purification of Anti-HPV16 E7 Polyclonal Antibodies}

To produce anti-HPV16 E7 polyclonal antibodies, New Zealand white rabbits were immunized by subcutaneous injection of about $500 \mu \mathrm{g}$ HPV16 E7 (in $0.8 \mathrm{ml}$ ) mixed with an equal volume of 
Freund's complete adjuvant (Sigma-Aldrich, USA). Booster injections were applied as described above but using Freund's incomplete adjuvant at 10-day intervals three times. The quality of the antibodies in sera was monitored by indirect enzyme-linked immunosorbent assay (ELISA), which was repeated daily until a threshold was determined $(1: 100,000)$.

For ELISA, as the antigen for ELISA, purified HPV16 E7 proteins were diluted to a final concentration of $50 \mu \mathrm{g} / \mathrm{ml}$ in $0.05 \mathrm{M}$ carbonate buffer solution $(\mathrm{pH} 9.6)$ at $37^{\circ} \mathrm{C}$ for one hour and at $4^{\circ} \mathrm{C}$ overnight. After washing with PBS containing 0.05\% Tween 20 and blocking with $200 \mu \mathrm{L} 5 \%$ milk at $37^{\circ} \mathrm{C}$ for 2 hours, $100 \mu \mathrm{L}$ anti-HPV16 E7 polyclonal antibody (1:100,000 diluted) was used as the primary antibody, and HRP-conjugated goat anti-rabbit antibody was used as the secondary antibody. TMB Solution was used as the color substrate and terminated with TMB Stop Solution, the OD450 was detected. Serums with the OD450 values higher than that of control serums by 2.1 -fold were defined as positive.

Another $500 \mu \mathrm{g}$ HPV16 E7 without adjuvant was injected ten days later. Rabbit sera were collected five days after the last immunization. The Protein G Agarose (Life Technology) was applied to purify polyclonal immunoglobulin according to the manufacturer's protocol. The serum was stored at $-20^{\circ} \mathrm{C}$ in aliquots.

\section{Western blotting analysis and Immunofluorescent}

For western blotting, cell lysates and protein samples were subjected to SDS-PAGE and transferred to PVDF membrane (Bio-Rad, USA). Membranes were blocked in PBS-T containing 5\% w/w non-fat milk (BD Difco ${ }^{\mathrm{TM}}$, USA). The rabbit polyclonal antibodies raised against HPV16 E7 were used as the primary antibodies. The peroxidase-conjugated anti-rabbit immunoglobulin G (IgG; diluted 1:5000 in PBS; Beyotime) was used as the secondary antibodies. Protein was detected with Enhanced Chemiluminescence (ECL) western blotting substrate (Millipore, USA) through a chemiluminescence imaging system (Fujifilm, USA).

For immunofluorescence, 293T cells and $\mathrm{SiHa}$ cells were fixed in $4 \%$ paraformaldehyde (Bio-Rad, USA) and permeabilized with $0.1 \%$ Triton X-100 (Solarbio, China). Anti-HPV16 E7 polyclonal antibody was used as the primary antibody and Alexa Fluor 488-conjugated donkey anti-rabbit IgG (1:500; Beyotime) was used as the secondary antibody. DAPI (Beyotime) was applied to stain the nucleus and stainings were observed under an immunofluorescence microscope (Olympus, USA).

\section{HPV16 E7 Immunohistochemistry}

Immunohistochemistry stains were performed as follows. Formalin-fixed paraffin-embedded tissue sections $(8 \mu \mathrm{m})$ were deparaffinized in xylene and rehydrated in graded ethanol. Heat-induced antigen retrieval was performed using citrate buffer ( $\mathrm{pH}$ 6.0). Slides were treated with $0.3 \%$ hydrogen peroxide for 30 minutes to quench endogenous peroxidase activity, blocked with PBS containing 10\% normal goat serum at room temperature for 30 minutes. Rabbit anti-HPV16 E7 polyclonal antibody (1:400) was used as the primary antibody and the horseradish peroxidase-conjugated goat anti-rabbit IgG antibody (1:1000) was applied as the secondary antibody. Slides were then incubated with DAB (3, 3'-diaminobenzidine) for 5 minutes, counterstained with hematoxylin for 5 seconds, visualized using a microscope (Carl Zeiss, Germany).

Five random high power fields $(400 \times)$ were observed to score the staining intensities and positive cell percentages. The staining intensities were quantified as follows: 0 (no staining); 1 (light brown); 2 (brown); 3 (dark brown). The positive cell percentages were graded as follows: $0(0 \%) ; 1(1-25 \%)$; $2 \quad(26-50 \%) ; 3 \quad(51-75 \%) ; 4 \quad(76-100 \%) . \quad$ The immunoreactive score (values, 0-12) was finally obtained by calculating the product of the staining intensity (0-3) and the positive cell percentage (0-4). According to the immunoreactive score, the staining results were defined as follows: negative, score $0-1$; low expression, score 2-3; moderate expression, score $4-5$; high expression, score $\geq 6$. The results were evaluated by two independent pathologists.

\section{Statistical analysis}

Spearman's rank correlation test was used for comparison between groups. All statistical analyses were performed using the SPSS 20.0 statistical software (SPSS Inc., Chicago, IL, USA). $P$ value $<0.05$ were considered statistically significant.

\section{Results}

\section{Expression of HPV16 E7 protein}

Firstly, The HPV16 E7 gene was inserted into the pGEX-4T2 vector. The recombinant pGEX-4T2(HPV16-E7) vectors were identified by gene sequencing and double enzyme digested with BamH I and EcoR I (Figure 1). Secondly, the vectors were transformed into Escherichia coli JM109 and induced with IPTG. Thirdly, the expressed HPV16 E7 fusion protein containing GST-tag was purified by Glutathione-Sepharose 4B beads, removed GST-tag by thrombin, and dialyzed overnight. Both fusion protein and purified protein were separated by $10 \%$ 
SDS-PAGE and visualized by Coomassie blue staining (Figure 2). It indicated that the molecular weight of the HPV16 E7 fusion protein was about 37 $\mathrm{kDa}$ and the purified protein was about $18 \mathrm{kDa}$.

\section{Expression of HPV16 E7 polyclonal antibody}

The HPV16 E7 polyclonal antibody was obtained from the rabbits that were immunized five times with purified HPV16 E7 protein. Rabbit serum antibody concentrate was monitored by ELISA and purified by the Protein G Agarose. The specificity of the purified anti-HPV16 E7 polyclonal antibody was verified by both western blotting and immunofluorescent stains (Figure 3). SiHa cells were known to continually expressing HPV16 E7 protein endogenously, and 293T cells were negative of HPV16 E7 protein. As shown in Figure 3, HPV16 E7 protein

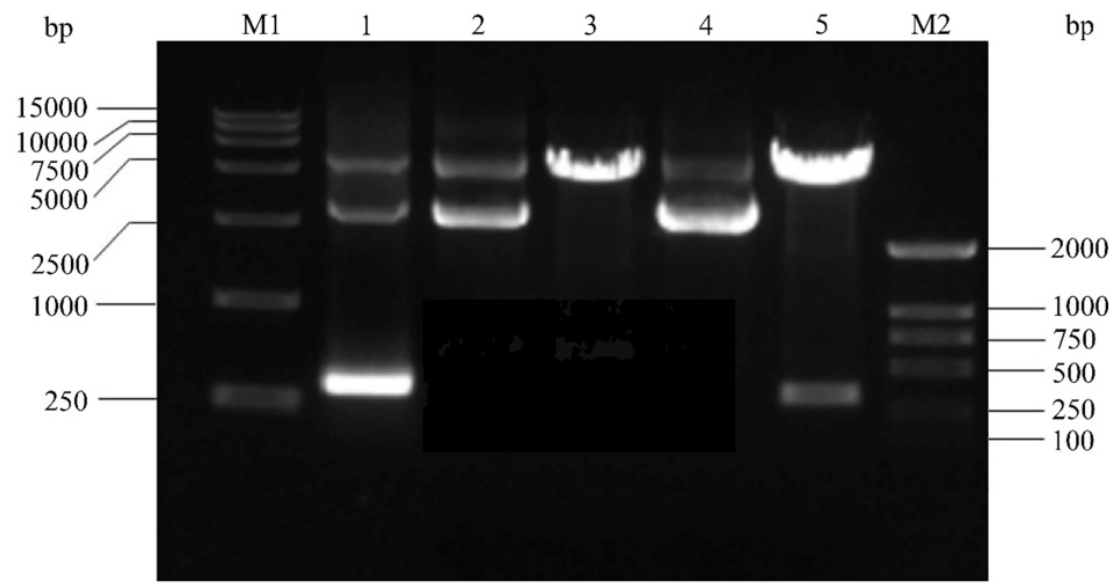

Figure 1. The construction of the HPV16 E7 prokaryotic expression vector. M1: DNA DL15000; Lane 1: Amplification product of HPV16 E7 by polymerase chain reaction (PCR) from HPV16 purified plasmid DNA; Lane 2: PGEX-4T2 vector; Lane 3: PGEX-4T2 vector was digested with the restriction enzymes of EcoR I and BamH I; Lane 4: recombinant plasmid PGEX-4T2-(HPV16-E7); Lane 5: recombinant plasmid was digested with the restriction enzymes of EcoR I and BamH I; M2: DNA DL2000. HPV, Human papillomavirus.
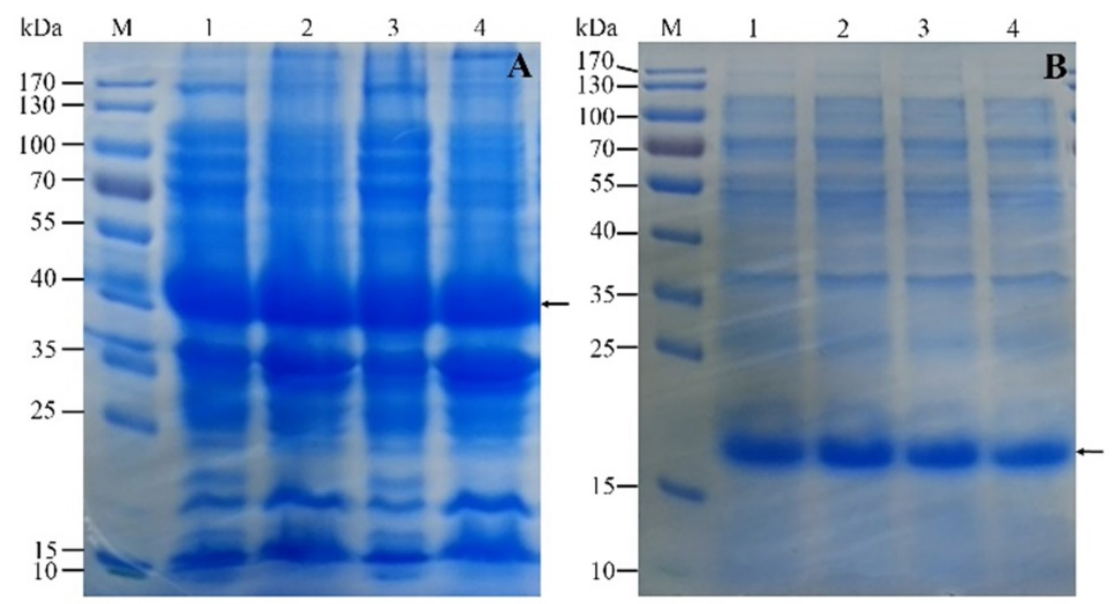

Figure 2. The expression and purification of recombinant HPV16 E7 protein. (A) Sodium salt-polyacrylamide gel electrophoresis (SDS-PAGE) analysis of the expression of the protein in JM109. M: protein marker; Lane 1 and 3: the supernatant of cells; Lane 2 and 4: the pellet of broken cells. Arrow indicates GST-(HPV16-E7) protein. (B) The SDS-PAGE analysis of the purification of the protein. M: protein marker; Lane 1 and 2: recombinant protein after removed GST-tag; Lane 3 and 4: the purified protein after dialyzed overnight. Arrow indicates HPV16 E7 protein. was detected by the homemade anti-HPV16 E7 polyclonal antibody in SiHa cell lysates but not in 293T cell lysates. Besides, our previous study proved that there is no cross-reaction of the polyclonal antibody between different HPV genotypes [11].

\section{Expression of HPV16 E7 protein in cervical}

Immunohistochemistry stains of HPV16 E7 protein were applied in a total amount of 182 of PCR proved HPV16 DNA-positive DNA-negative chronic cervicitis tissue specimens (Figure 4). It also indicated that the HPV16 E7 protein was localized in the nucleus. As shown in Figure 5, the positive rate of HPV16 E7 protein expression was elevated with increasing either histological or cytological malignancy grade. It also showed a positive correlation between HPV16 E7 protein expression positive rates and histological grade $(\mathrm{r}=0.675, p<$ 0.01), HPV16 E7 protein expression levels and histological grade $(\mathrm{r}=0.756, p<0.01)$, and HPV16 E7 protein expression positive rates with cytological malignancy grade $(\mathrm{r}=1, p<0.01)$ (Table 1 and 2). Since all 182 samples were PCR proved HPV16 DNA-positive, the positive coincidence rate of HPV16 E7 protein immunohistochemistry stain compared to HPV genotyping analysis in cervical intraepithelial neoplasia 1 (CIN1), CIN2, CIN3, and cervical cancer was equal to HPV16 E7 protein expression positive rates as $15 \%, 93 \%, 100 \%$, and $100 \%$. There is a positive correlation between HPV16 E7 protein immunohistochemistry stain and HPV genotyping analysis in positive coincidence rate $(\mathrm{r}=0.675, p<0.01)$. The negative coincidence rate was $100 \%$ accordingly. However, for the different tumor sizes, tumor differentiation, lymph node metastasis, International Federation of Gynecology and Obstetrics (FIGO) stage, or lymphovascular space invasion in 58 cases of cervical cancer, there is no relationship between these subgroups in HPV16 E7 protein expression levels (Table 3) $(p>0.05$, respectively). 
A

HPV-16E7 SiHa

293T

Anti-16E7

B

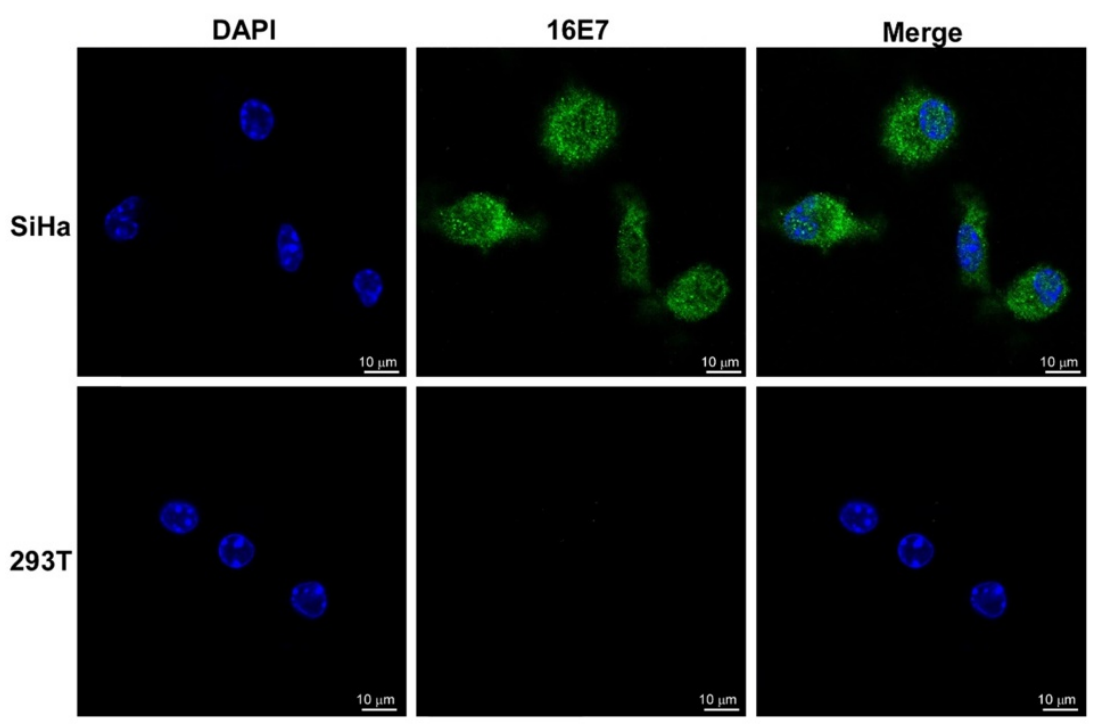

Figure 3. The specificity of the purified anti-HPV16 E7 polyclonal antibody. (A) Western blot analysis. HPV16 E7: purified HPV16 E7 protein; 293 T: 293 T cells lysates; SiHa: SiHa cells lysates. (B) Immunofluorescence stains. Photomicrographs showed the distribution of immunoreactive HPV16 E7 protein (green) and DAPI-stained nucleus (blue) in SiHa cells and 293 T cells.

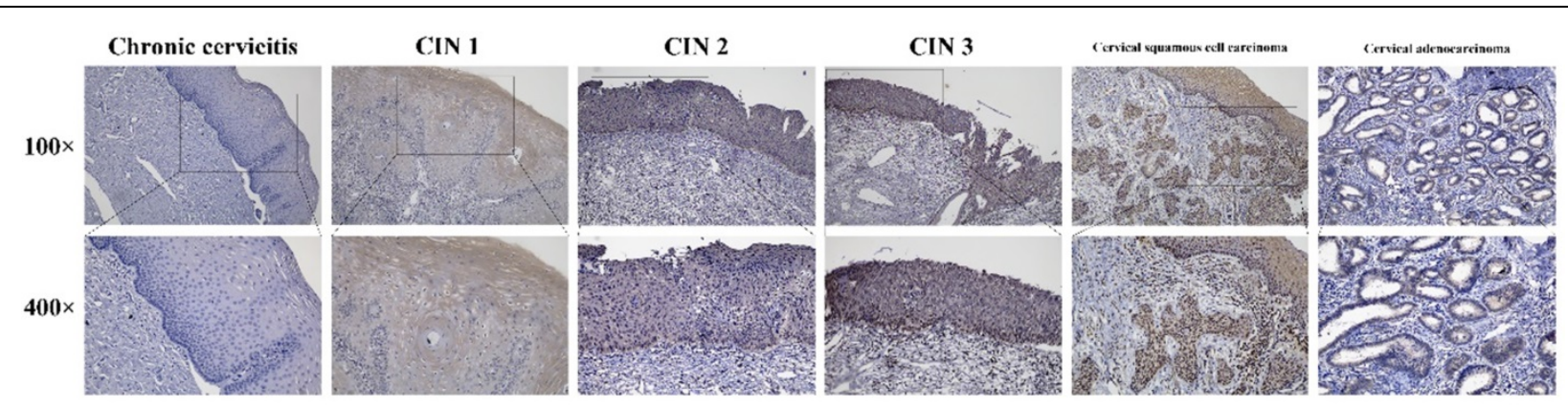

Figure 4. Immunohistochemistry stains of the HPV16 E7 protein in various cervical lesions. The purified HPV16 E7 antibody was used as a primary antibody in chronic cervicitis, cervical intraepithelial neoplasia (CIN) 1, CIN 2, CIN 3, cervical squamous cell carcinoma, and cervical adenocarcinoma tissue specimens. The brown color indicates positive stain.

Table 1. HPV16 E7 protein expression in various cervical tissue specimens.

\begin{tabular}{|c|c|c|c|c|c|c|}
\hline \multirow[t]{2}{*}{ Histology } & \multicolumn{4}{|c|}{ HPV16 E7 protein expression levels, $\mathrm{N}$} & \multirow[t]{2}{*}{ Total, $\mathrm{N}$} & \multirow[t]{2}{*}{ Positive Rate (\%) } \\
\hline & Negative & Low & Moderate & High & & \\
\hline Chronic cervicitis & 33 & 0 & 0 & 0 & 33 & 0 \\
\hline Cervical Intraepithelial Neoplasia 1 (CIN 1) & 34 & 5 & 1 & 0 & 40 & 15 \\
\hline Cervical Intraepithelial Neoplasia 2 (CIN 2) & 3 & 2 & 10 & 28 & 43 & 93 \\
\hline Cervical Intraepithelial Neoplasia 3 (CIN 3) & 0 & 1 & 2 & 35 & 38 & 100 \\
\hline Cervical cancer & 0 & 0 & 2 & 59 & 61 & 100 \\
\hline
\end{tabular}

HPV16 E7 protein expression levels were classified into four groups (negative, low, moderate, and high) with relative staining levels.

Table 2. HPV16 E7 protein expression in various cervical cytological specimens.

\begin{tabular}{|c|c|c|c|c|}
\hline \multirow[t]{2}{*}{ Cytology } & \multicolumn{2}{|c|}{ HPV16 E7 protein-positive, $\mathrm{N}$} & \multirow[t]{2}{*}{ Total, $\mathrm{N}$} & \multirow{2}{*}{$\begin{array}{l}\text { Positive Rate } \\
(\%)\end{array}$} \\
\hline & Negative & Positive & & \\
\hline Negative for Intraepithelial Lesion or Malignancy (NILM) & 41 & 20 & 61 & 67.2 \\
\hline Atypical Squamous Cells of Undetermined Significance (ASC-US) & 21 & 8 & 29 & 72.4 \\
\hline Low-grade Squamous Intraepithelial Lesion (LSIL) & 14 & 3 & 17 & 82.4 \\
\hline Atypical Squamous Cells, which cannot exclude a High-grade lesion (ASC-H) & 20 & 2 & 22 & 90.9 \\
\hline High-grade Squamous Intraepithelial Lesion (HSIL) & 49 & 4 & 53 & 92.5 \\
\hline
\end{tabular}



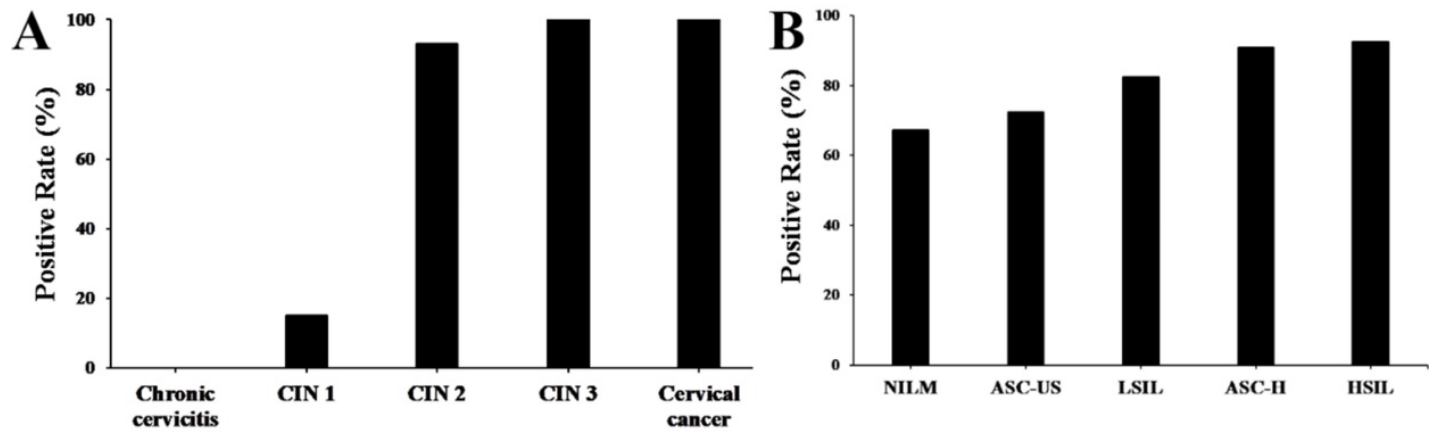

Figure 5. The positive rate of HPV16 E7 protein in various cervical lesions. (A) The positive rate of HPV16 E7 protein in cervical lesions with various histological grades according to the FIGO staging systems. (B) The positive rate of HPV16 E7 protein in cervical lesions with different cytological grades according to the Bethesda systems.

Table 3. HPV16 E7 protein expression in cervical cancer.

\begin{tabular}{|c|c|c|c|c|}
\hline Variables & Total & $\begin{array}{l}\text { Moderate HPV16 E7 } \\
\text { protein expression, } \\
\mathrm{N}\end{array}$ & $\begin{array}{l}\text { High HPV16 E7 } \\
\text { protein expression, } \\
\mathrm{N}\end{array}$ & $P$ value \\
\hline \multicolumn{5}{|l|}{ Tumor size, $\mathrm{cm}$} \\
\hline$<4$ & 58 & 2 & 56 & $\mathrm{r}=0.042$, \\
\hline$\geq 4$ & 3 & 0 & 3 & $p>0.05$ \\
\hline \multicolumn{5}{|l|}{$\begin{array}{l}\text { Degree of } \\
\text { differentiation }\end{array}$} \\
\hline Well & 19 & 0 & 19 & $\mathrm{r}=0.124$, \\
\hline Moderate and poor & 42 & 2 & 40 & $p>0.05$ \\
\hline \multicolumn{5}{|c|}{ Lymph node metastasis } \\
\hline No & 54 & 2 & 52 & $\mathrm{r}=0.066$, \\
\hline N1 & 7 & 0 & 7 & $p>0.05$ \\
\hline \multicolumn{5}{|l|}{ FIGO stage } \\
\hline I and II & 54 & 2 & 52 & $\mathrm{r}=0.066$, \\
\hline III & 7 & 0 & 7 & $p>0.05$ \\
\hline \multicolumn{5}{|c|}{$\begin{array}{l}\text { Lymphovascular space } \\
\text { invasion (LVSI) }\end{array}$} \\
\hline Negative & 54 & 2 & 52 & $\mathrm{r}=0.066$, \\
\hline Positive & 7 & 0 & 7 & $p>0.05$ \\
\hline
\end{tabular}

\section{Discussion}

The HPV infection is associated with a broad spectrum of diseases that range from self-limiting skin warts to life-threatening cancers. The hr-HPV has been confirmed with close links to invasive cervical cancer, especially HPV16 and 18. Moreover, E7 protein encoded by hr-HPV types plays a crucial role in carcinogenesis by the following mechanism: Initially, E7 oncoproteins might down-regulate TLR-9, a fundamental role in pathogen recognition and innate immune activation, to evade the immune response and cause persistent infection [12]. Consequently, E7 oncoproteins could degrade tumor suppressor proteins p53 and retinoblastoma protein and inhibit cyclin dependent kinase inhibitors (p21, p27, p16) to disrupt cell cycle checkpoint control, which results in host cells proliferation without DNA damage repaired. Besides, E7 could bind to STING, an adaptor protein downstream of the intracellular DNA sensor via the LXCXE motif, to suppress a post-infection immune response [13]. E7 could also bind to and augment c-Myc-mediated transcription activity, which Myc is inarguably the most potent oncogene in the human cell, to induce uncontrolled cell growth and proliferation [14]. Additionally, E7 could cause DNA damage and chromosomal aberrations due to replication stress. Silencing of E7 revealed a significant decrease in DNA damage [15]. Furthermore, E7 could induce abnormal centrosome synthesis, which is another mechanism of genomic instability in malignant tumors, leading to aneuploidy [16]. In the epigenetic regulation, E7 could upregulate DNA methyltransferases DNMT1, DNMT3A, and DNMT3B to promote genomic instability [17], induce methylation of CXCL14 to promote tumor growth via angiogenesis [18]. Therefore, the antibody against HPV16 E7 proteins could be a valuable tool for better understands the crucial role of HPV16 E7 protein in carcinogenesis both experimentally and clinically.

There are various methods to detect HPV16 E7 protein, from detecting antibodies to HPV16 E7 protein in serum by radioimmunoprecipitation assay [19], ELISA [20], immunoassay platform [21] or fluorescence detection platform [22], to directly detect HPV16 E7 protein by immunohistochemistry study via polyclonal [23] or monoclonal [24] antibody. Compared to indirect antibodies detection, the immunohistochemistry study could directly and visually demonstrate HPV16 E7 oncoproteins are present. The increasing positive coincidence rate with malignancy grade and $100 \%$ negative coincidence rate between HPV genotyping and immunohistochemistry also indicate that the immunohistochemistry study is more specific in detecting persistent infection rather than transient infection, especially in early cervical cancer. Besides, it could prevent excess diagnosis or therapy to reduce the public health burden. Compare to monoclonal antibodies, polyclonal antibodies were considered a higher sensitivity in the application and less procedure in preparation. Furthermore, immunohistochemistry studies with lower costs and easier operation are especially fit for the transitioning economies without sufficient skilled pathologists. In this study, we have prepared a repeatable, accessible, 
productive, sensitive and low-cost anti-HPV16 E7 polyclonal antibody.

Previous studies indicate that both HPV16 E7 mRNA [25] and protein [26] expression is higher in women with HISL than LISL, a severe cervical dysplasia than a slight cervical dysplasia. It is similar to our result in comparing the HPV16 E7 protein-positive rate with histology and cytological malignancy grades indicated that HPV16 E7 oncoprotein could be a useful biomarker for identifying persistent HPV infection from malignant and pre-malignant lesions. In summary, these advantages noted that our homemade HPV16 E7 polyclonal antibody could be a valuable tool to evaluate the severity of cervical lesions clinically with higher specificity, higher sensitivity, visuality, more economically and easier operation, especially in low/medium HDI regions. However, in our study, there is no relationship between HPV16 E7 protein expression levels and tumor sizes, tumor differentiation, lymph node metastasis, FIGO stage or lymphovascular space invasion. It might contribute to a variety of confounding biases and suggest the HPV16 E7 protein plays a crucial role in tumorigenesis rather than a prognosis marker. For the limitation, our study applied the antibody on cervical tissue specimens rather than cytological samples make it hard to evaluate its diagnosis role. Further research focuses on early screening or diagnosis could better illustrate the value of our polyclonal antibody.

In conclusion, the present study demonstrates the preparation of HPV16 E7 polyclonal antibody and a reliable immunohistochemistry protocol for its application in various cervical lesions. The positive coincidence rate of HPV16 E7 protein immunohistochemistry stain compared to HPV genotyping analysis in CIN1, CIN2, CIN3, and cervical cancer was $15 \%, 93 \%, 100 \%$, and $100 \%$, while the negative coincidence rate was $100 \%$ accordingly. It revealed an elevated HPV16 E7 protein-positive rate in HPV16 DNA-positive cervical lesions with increasing histological and cytological malignancy grades. However, there is no relationship between HPV16 E7 protein expression levels and tumor sizes, tumor differentiation, lymph node metastasis, FIGO stage or lymphovascular space invasion in cervical cancer. The preparation of the HPV16 E7 protein in vitro may provide a basis for further research focusing on the role of HPV E7 proteins in various cancers.

\section{Acknowledgements}

This study was supported by grants from the National Natural Science Foundation of China (Grant No. 81573057, 81472889 and 82103743), the National Health Commission Research Foundation of China
(Grant No. 2015117502), the Science and Technology Projects of Zhejiang Province (Grant No. 2018C04013) and the Natural Science Foundation of Zhejiang Province (Grant No. LQ19H190002).

\section{Compliance with Ethics Guidelines}

\section{Animal and Human Rights Statement}

All institutional and national guidelines for the care and use of laboratory animals were followed. Additional informed consent was obtained from all patients for whom identifying information is included in this article.

\section{Author Contributions}

JZ proposed the study and wrote the first draft. LC collected and analyzed the data. All authors contributed to the intellectual content and approved the final manuscript, and $\mathrm{HC}$ is the guarantor.

\section{Competing Interests}

The authors have declared that no competing interest exists.

\section{References}

1. Bray F, Ferlay J, Soerjomataram I, Siegel RL, Torre LA, Jemal A. Global cancer statistics 2018: GLOBOCAN estimates of incidence and mortality worldwide for 36 cancers in 185 countries. CA: a cancer journal for clinicians. 2018; 68: 394-424.

2. Walboomers JM, Jacobs MV, Manos MM, Bosch FX, Kummer JA, Shah KV, et al. Human papillomavirus is a necessary cause of invasive cervical cancer worldwide. The Journal of pathology. 1999; 189: 12-9.

3. zur Hausen H. Papillomaviruses causing cancer: evasion from host-cell control in early events in carcinogenesis. Journal of the National Cancer Institute. 2000; 92: 690-8.

4. Halbert CL, Demers GW, Galloway DA. The E7 gene of human papillomavirus type 16 is sufficient for immortalization of human epithelial cells. Journal of virology. 1991; 65: 473-8.

5. Flores ER, Allen-Hoffmann BL, Lee D, Lambert PF. The human papillomavirus type $16 \mathrm{E} 7$ oncogene is required for the productive stage of the viral life cycle. Journal of virology. 2000; 74: 6622-31.

6. Morris JD, Crook T, Bandara LR, Davies R, LaThangue NB, Vousden KH. Human papillomavirus type $16 \mathrm{E} 7$ regulates E2F and contributes to mitogenic signalling. Oncogene. 1993; 8: 893-8.

7. Barnard P, Payne E, McMillan NA. The human papillomavirus E7 protein is able to inhibit the antiviral and anti-growth functions of interferon-alpha. Virology. 2000; 277: 411-9.

8. Dyson N, Howley PM, Munger K, Harlow E. The human papilloma virus-16 E7 oncoprotein is able to bind to the retinoblastoma gene product. Science. 1989; 243: 934-7.

9. Spitkovsky D, Hehner SP, Hofmann TG, Moller A, Schmitz ML. The human papillomavirus oncoprotein E7 attenuates NF-kappa B activation by targeting the Ikappa B kinase complex. The Journal of biological chemistry. 2002; 277: 25576-82.

10. Ronco G, Dillner J, Elfstrom KM, Tunesi S, Snijders PJ, Arbyn M, et al. Efficacy of HPV-based screening for prevention of invasive cervical cancer: follow-up of four European randomised controlled trials. Lancet. 2014; 383: 524-32.

11. Zheng Q, Wang T, Jiang S, Han R, Jin N, Zhu J, et al. Production of Polyclonal Antibody to the HPV58 E7 Protein and Its Detection in Cervical Cancer. PloS one. 2016; 11: e0169138.

12. Hasan UA, Bates E, Takeshita F, Biliato A, Accardi R, Bouvard V, et al. TLR9 expression and function is abolished by the cervical cancer-associated human papillomavirus type 16. Journal of immunology. 2007; 178: 3186-97.

13. Lau L, Gray EE, Brunette RL, Stetson DB. DNA tumor virus oncogenes antagonize the cGAS-STING DNA-sensing pathway. Science. 2015; 350: 568-71.

14. Wang YW, Chang HS, Lin CH, Yu WC. HPV-18 E7 conjugates to c-Myc and mediates its transcriptional activity. The international journal of biochemistry \& cell biology. 2007; 39: 402-12.

15. Cortes Gutierrez EI, Garcia-Vielma C, Aguilar-Lemarroy A, Vallejo-Ruiz V, Pina-Sanchez P, Zapata-Benavides P, et al. Expression of the HPV18/E6 
oncoprotein induces DNA damage. European journal of histochemistry : EJH. 2017; 61: 2773

16. Duensing S, Duensing A, Crum CP, Munger K. Human papillomavirus type $16 \mathrm{E} 7$ oncoprotein-induced abnormal centrosome synthesis is an early event in the evolving malignant phenotype. Cancer research. 2001; 61: 2356-60.

17. Burgers WA, Blanchon L, Pradhan S, de Launoit $Y$, Kouzarides T, Fuks F. Viral oncoproteins target the DNA methyltransferases. Oncogene. 2007; 26: 1650-5.

18. Cicchini L, Westrich JA, Xu T, Vermeer DW, Berger JN, Clambey ET, et al. Suppression of Antitumor Immune Responses by Human Papillomavirus through Epigenetic Downregulation of CXCL14. mBio. 2016; 7.

19. Stacey SN, Ghosh A, Bartholomew JS, Tindle RW, Stern PL, Mackett M, et al. Expression of human papillomavirus type 16 E7 protein by recombinant baculovirus and use for the detection of E7 antibodies in sera from cervical carcinoma patients. Journal of medical virology. 1993; 40: 14-21.

20. Ramezani A, Aghakhani A, Soleymani S, Bavand A, Bolhassani A. Significance of serum antibodies against HPV E7, Hsp27, Hsp20 and Hp91 in Iranian HPV-exposed women. BMC infectious diseases. 2019; 19: 142.

21. Inan $\mathrm{H}$, Wang $\mathrm{S}$, Inci $\mathrm{F}$, Baday $\mathrm{M}$, Zangar $\mathrm{R}$, Kesiraju $\mathrm{S}$, et al. Isolation, Detection, and Quantification of Cancer Biomarkers in HPV-Associated Malignancies. Scientific reports. 2017; 7: 3322.

22. Obahiagbon U, Smith JT, Zhu M, Katchman BA, Arafa H, Anderson KS, et al. A compact, low-cost, quantitative and multiplexed fluorescence detection platform for point-of-care applications. Biosensors \& bioelectronics. 2018; 117: $153-60$

23. Ressler S, Scheiden R, Dreier K, Laich A, Muller-Holzner E, Pircher H, et al. High-risk human papillomavirus E7 oncoprotein detection in cervical squamous cell carcinoma. Clinical cancer research : an official journal of the American Association for Cancer Research. 2007; 13: 7067-72.

24. Dreier K, Scheiden R, Lener B, Ehehalt D, Pircher H, Muller-Holzner E, et al. Subcellular localization of the human papillomavirus $16 \mathrm{E} 7$ oncoprotein in CaSki cells and its detection in cervical adenocarcinoma and adenocarcinoma in situ. Virology. 2011; 409: 54-68,

25. Bruno MT, Ferrara M, Fava V, Rapisarda A, Coco A. HPV genotype determination and E6/E7 mRNA detection for management of HPV positive women. Virology journal. 2018; 15: 52 .

26. Lee HJ, Kim JW. Immunohistochemical study on the expression of matrix metalloproteinase 2 and high-risk human papilloma virus in the malignant progression of papillomas. Journal of the Korean Association of Oral and Maxillofacial Surgeons. 2013; 39: 224-30 